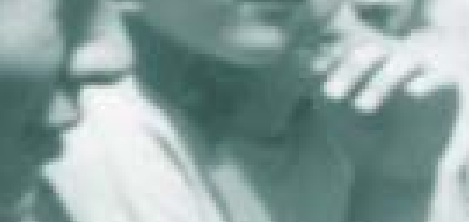

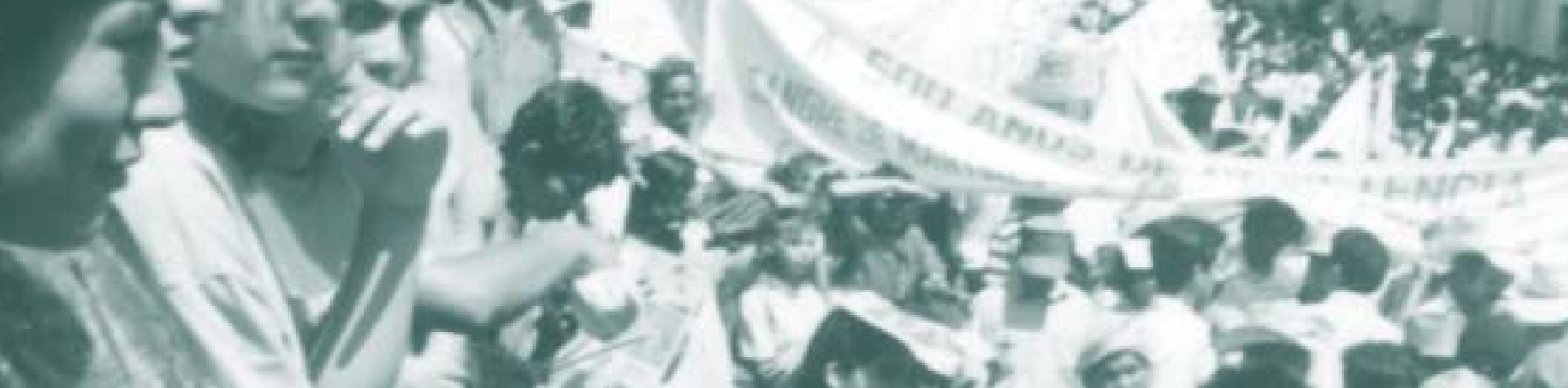

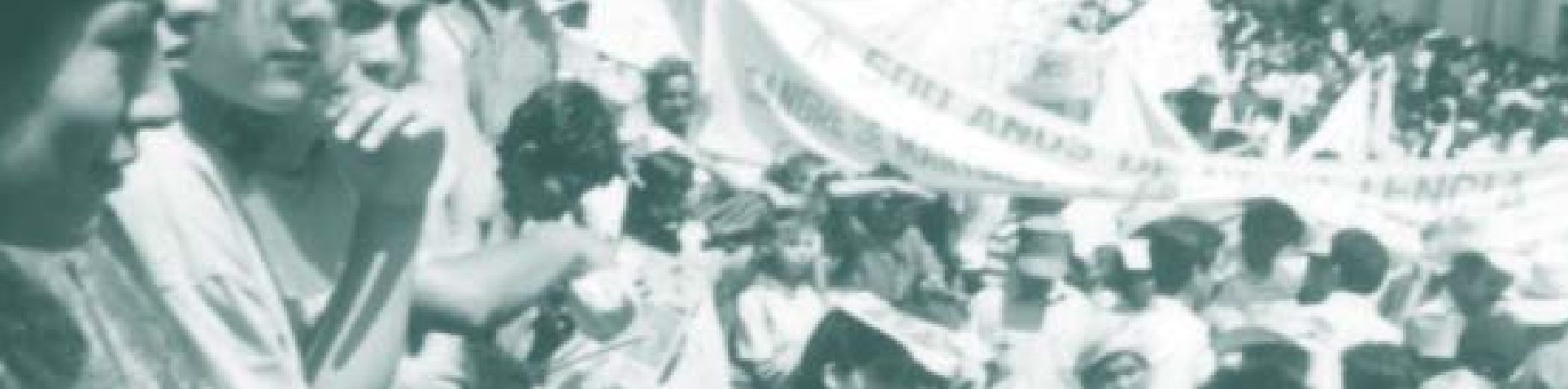

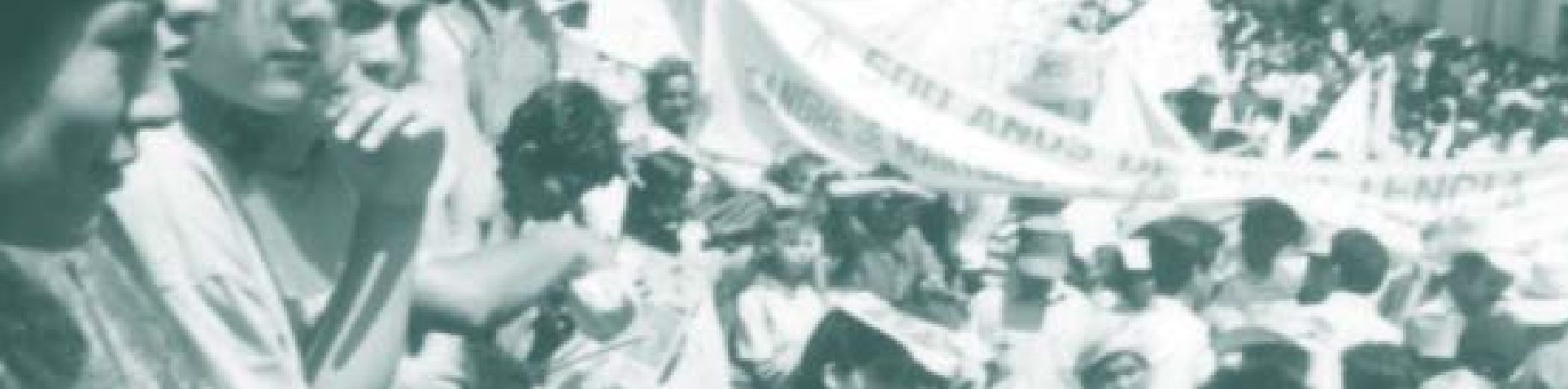

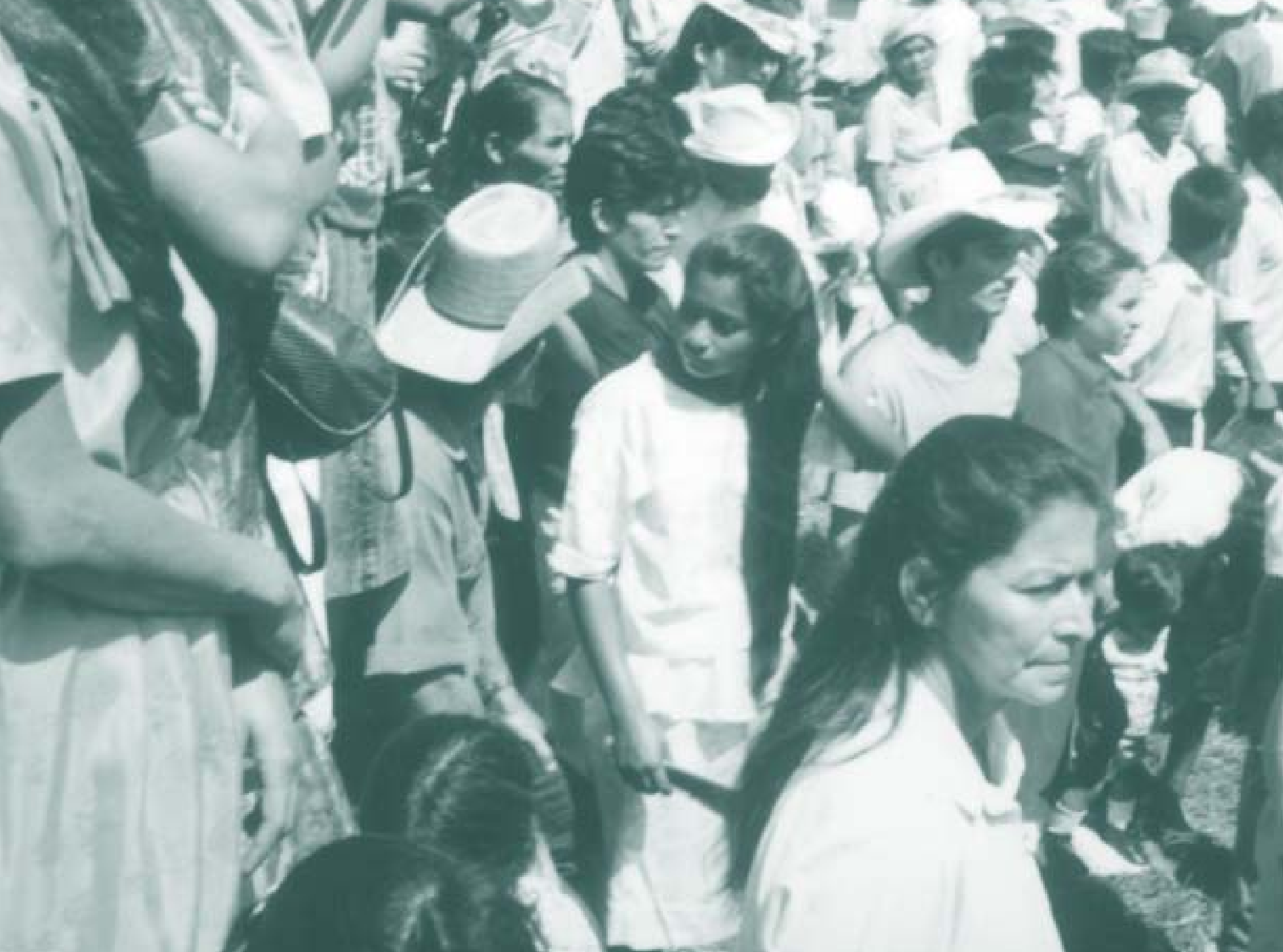




\title{
3 las encuestas sociodemográficas en Honduras
}

\author{
David Alexander Figueroa Toruño \\ Elvis Geovany Trejo Teruel
}

\section{INTRODUCCIÓN}

Las encuestas son instrumentos necesarios para recopilar información valiosa en diferentes áreas de la investigación y nos dan una visión más profunda, periódica, actualizada y detallada de los hechos demográficos.

Este trabajo busca, de al guna manera, sistematizar la información que se puede obtener de las múltiples encuestas de carácter demográfico realizadas en Honduras, Ias cuales enmarcan y analizan varios aspectos: sociodemográficos, salud, educación, planificación familiar, economía, agrícolas y otros de carácter mixto.

El principal propósito de este documento es caracterizar las encuestas demográficas realizadas en $\mathrm{H}$ onduras durante el período 1962- 2002, donde se analizan las variables que contienen Ias distintas encuestas aplicadas en el país y la problemática existente en la evolución de las mismas, tomando en cuenta sus ventajas, desventajas, errores, problemas y evolución de las mismas.

Para conocer más sobre cada una de las encuestas se recurrió a lasinstituciones y organismos encargados del diseño y aplicación de Ias mismas como el Instituto $\mathrm{N}$ acional de Estadísticas (INE), A SHON PLA FA, alcaldías entre otras.

\section{Tipos de encuestas demográficas}

U na encuesta se define "como una operación mediante el cual se obtiene información detallada sobre las características de una población a partir de la observación de una parte o subconjunto de ella" (Banguero, 2001).

Las en cuestas "son utilizadas como fuentes de datos para análisis demográficos referidos, básicamente, a los componentes del cambio de la población", habitualmente recogen información a nivel de cada individuo, pero también consideran criterios para definir hogares (Welti, $1997: 43$ ).

Los temas incluidos en las encuestas demográficas se clasifican en cinco grandes grupos:
1. Características demográficas generales tales como sexo y edad de la población.

2. La fecundidad y sus determinantes.

3. La mortalidad infantil, materna y adulta.

4. Migración y movilidad.

5. Características socioeconómicas rel evantes.

Según W elti, 1997 :43 las encuestas demográficas pueden ser de dos tipos:

1. Encuestas prospectivas

- Registran los movimientos y hechos demográficos de la población bajo observación durante un período de tiempo.

- Se aplica un cuestionario de manera reiterada a una misma persona o grupo de personas dentro de un hogar durante el período de estudio.

- A través del formulario se recogen datos y se registran los principales hechos demográficos (nacimientos, defunciones, enfermedades, matrimonios, migraciones) que ocurren en un lapso definido.

\section{Encuestas retrospectivas}

- Se trata de un cuestionario que se aplica en una sola ocasión.

- Las repuestas permiten reconstruir la historia demográfica de los individuos entrevistados.

- Se utilizan para la mortalidad y la fecundidad, preguntas que permiten la estimación directa e indirecta de ambas variables.

- En el caso de la migración, normalmente las preguntas indagan la historia de cambios de residencia de los individuos y las motivaciones para salir del lugar de origen y elegir el de destino.

$\mathrm{N}$ o cabe duda que existen una serie de ventajas en la aplicación de las encuestas en cuanto a la información que registran. Sin embargo, existen también una serie de desventajasy de errores en la información obtenida en las encuesta, tal como puede verse en el siguiente cuadro. 
- La principal ventaja es que al usar el muestreo implica menor costo que un censo.

- Contiene una mayor cantidad de preguntas especificas que el censo.

- Se pueden hacer estudios demográficos de mayor profundidad.

- Se realizan más periódicamente que los censos por lo tanto se obtiene una información actualizada de los cambios demográficos.

- Sirven para diseñar políticas y programas dirigidos a la población, de acuerdo a la evolución de esta.
- La principal desventaja consiste en los errores de muestreo.

- No cubren el total de una población.

- Debido a su periodicidad representan costos en su aplicación.
- Los errores más frecuentes provienen del muestreo (deficiencia en el marco muestral provocando omisiones).

- A menudo se producen diseños muestrales mal definidos.

- Errores de contenido, como la mala elaboración del cuestionario.

- En ocasiones se dan errores en la codificación o digitación.

Fuente: Elaboración propia y datos de Welti, 1997: 43-46.

\section{Encuestas aplicadas en Honduras}

U no de los mejores estudios sobre las encuestas demográficas en $\mathrm{H}$ onduras es de Ralph $\mathrm{H}$ acker en su documento La Población de H onduras: U na Síntesis ( páginas 48-58), sien do éste la base principal en esta investigación, otras fuentes consultadas son citadas oportunamente.

Durante los últimos años se realizaron un importante número de encuestas en el país, para completar la información recogida en los censos. Por sus objetivos primordiales, dichas encuestas pueden ser divididas en cinco categorías:

1. Encuestas demográficas generales.

2. Encuestas de salud y planificación familiar.

3. Encuestas de nutrición.

4. Encuestas sobre la fuerza de trabajo y economía doméstica.

5. Encuestas agrícolas.

A Igunas encuestas tienen características mixtas, la Encuesta de Líderes $\mathrm{N}$ acionales no clasifica en ninguna categoría.

A continuación se describe la evolución de esta tipología de encuestas:

\section{Encuesta Demográfica Nacional de Honduras (EDEN H)}

- La EDEN H I (1970-72) fue la primera encuesta demográfica general levantada en Honduras.

- Se trató de una encuesta de visitas repetidas, que tenían como objetivo disponer de un conjunto de tasas demográficas confiables que permitan evaluar y conocer la situación actual de los niveles de mortalidad, fecundidad, nupcialidad y las migraciones en $\mathrm{H}$ onduras.

- La muestra consistió de 6,470 viviendas (2,172 urbanasy 4,298 rurales).

- También se incorporó un cuestionario de preguntas retrospectivas (RETROEDEN H) con el propósito de comprobar la exactitud de al gunos resultados de la EDEN H y estudiar los métodos retrospectivos e indirectos que se ensayaban.

- $\quad$ En 1983 se realizó la EDEN H II, con el fin de mejorar y actualizar el conocimiento de las características demográficas de la población.

- La EDENH II se realizó con 11,106 viviendas.

- U na ventaja de esta encuesta es que se investigó con mayor profundidad y precisión la magnitud de las características económicas de la población femenina.

- Otra innovación fue el estudio de la migración internacional, mediante la inclusión de preguntas sobre familiares residentes en el exterior.

O bjetivos de la EDEN H II:

1. O btener estimaciones sobre la fecundidad actual y del pasado reciente para el total del país.

2. Estimaciones sobre la mortalidad de la niñez y en las edades adultas.

3. M igración de hondureños hacia el exterior.

4. Migraciones internas y características de la población migrante.

5. Estudiar la actividad económica de la mujer. 


\section{Encuesta Estándar de Población e Indicadores Socioeconómicos (EPIS)}

- Realizada por primera vez en San Pedro Sula en 1991 luego en los años 94 y 95, para obtener datos sobre la situación demográfica social de este municipio.

- Fue elaborada por la U nidad de Investigación Estadística Social de 1991-1995, con muestras de 3,000-4,000 hogares del área urbana.

- El diseño usado en estas encuestas es el mismo utilizado por las encuestas $\mathrm{N}$ acionales de H ogar Salud y Planificación Familiar, con la salvedad que las preguntas son más detalladas.

- METROPLA N también realizó al gunas encuestas en el Distrito $C$ entral, como la de los barrios marginados, en 1978 recolectó información sobre 7,479 viviendas en 34 barrios de la capital.

- Este tipo de Encuestas a nivel municipal también se han realizado en otras ciudades: Puerto Cortés y Santa Rosa de Copán en 1995, Progreso y Comayagua en 1996, el muestreo utilizado fue de tipo probabilístico.

- Estas municipalidades realizaron estas encuestas con el apoyo técnico y financiero del Fondo de Población de las $\mathrm{N}$ aciones U nidas (FN U A P) a través de proyectos dedicados a promover información sociodemográfica para el desarrollo municipal" (Entrevista, Plata Jorge: 2002).

- Estas encuestas proporcionan información referente a viviendas, servicios básicos, migración, educación, participación laboral, ingresos, mortalidad infanto-juvenil, conocimiento sobreel SIDA y uso de anticonceptivos (EPIS, Comayagua, 1998).

\section{Encuesta Nacional de Prevalencia del U so de Anticonceptivos (EN PA)}

- Realizada en 1981, la finalidad era estimar la magnitud del conocimiento, disponibilidad y uso de métodos anticonceptivos de la población hondureña.

- La muestra fue de 4,914 hogares a nivel nacional, se entrevistaron 3,968 mujeres de 15-49 años.

- Proporciona información sobre edad, estado civil, nivel de instrucción, ocupación, fecundidad, preferencias, actitudes reproductivas de mujeres en unión conyugal, conocimientos y disponibilidad de métodos anticonceptivos.

\section{Encuesta Nacional de Salud Materno Infatil (EN SMI)}

- Se realizó a nivel nacional en 1984, se visitaron 5,771 viviendas en las que se entrevistaron 5,454 mujeres entre 14 y 44 años de edad.

- Dirigida a recolectar y analizar información sobre fecundidad, mortalidad infantil, lactancia materna, atención por parteras tradicionales, inmunización, diarreas, planificación familiar, agua, disposición de excretas, instalaciones eléctricas.

- Se diferencia de las anteriores en que le da mayor atención al saneamiento básico de las vivien das consultadas, acción de parteras tradicionales, el puerperio y las diarreas.

\section{Encuesta Nacional de Epidemiología y Salud Familiar (ENESF 1987, 1991-92, 1996, 2001)}

- La primera se realizó entre junio y noviembre de 1987 se evaluaron un total de 11,732 viviendas de Tegucigal pa, San Pedro Sula y otros poblados de más de 2,000 habitantes, así como también el área rural, la muestra abarcó un total de 11,233 mujeres entre 15 y 44 años. El mayor peso se le asignó a las dos principales ciudades.

- La segunda se llevó a cabo entre 1991-92 la muestra fue de 7,635 viviendas, incluyen do los mismos lugares con 8,239 mujeres.

- La tercera se hizo en 1996 incluyo 9,647 hogares con 7,505 mujeres entrevistadas y por primera vez se entrevistaron 2,925 hombres sobre conocimientos y actitudes referentes al SIDA y ETS.

- La encuesta de 1987 tiene el mérito de ser una de las pocas fuentes de datos que permite el análisis de la migración interna.

- U na desventaja de la encuesta EN ESF 1991-92, es que limitó la historia de nacimiento a los hijos nacidos durante los últimos cinco años antes de la encuesta, limitando la estimación de los intervalos ínter genésicos, corrigiéndose el problema en 1996 al tomar las historias completas de nacimiento. En esta encuesta se diseñaron dos tipos de cuestionario:

- Dela vivienda.

0 Individual orientado a mujeres de 15-44 años.

- Estos estudios son de tipo descriptivo - transversal a nivel nacional sin contar con los departamentos de las I slas de la Bahía y Gracias A Dios.

\section{ANTECEDENTES DE LA ENCUESTA ENESF/ENSM 2001}

Según el informe resumido de la Encuesta $\mathrm{N}$ acional de Epidemiología y Salud Familliar (EN ESF 2001) y Encuesta N acional de Salud M asculina EN SM -2001 (2001:3). La EN ESF 2001 es la séptima de una serie de encuestas que desde 1981 se han real izado en $\mathrm{H}$ onduras para determinar los diferenciales y las ten dencias de la mortalidad y fecundidad.

El propósito es mantener una evaluación periódica de la evolución de las condiciones de salud reproductiva, en esta encuesta, han sido incluidos nuevos módulos como nutrición, anemia en los niños y sus madres, escolaridad de los niños de 5 a 14 años de edad.

En esta encuesta se incluyó un apartado titulado EN SM 2001 referido a establecer una Encuesta $\mathrm{N}$ acional de $\mathrm{H}$ ombres de edad reproductiva, sien do la primera realizada en 1996, con el propósito de determinar sus actitudes y comportamientos con respecto a la salud en general, salud sexual y reproductiva. 
Los resultados de las encuestas sirven para ayudar en el diseño de nuevas políticas y programas, reestructurar las actuales, proporcionar datos de línea de base para dar seguimiento a los procesos y nuevas iniciativas para evaluar su impacto.

El tamaño de la muestra de la EN ESF -01 es de 12,000 viviendas distribuidas en 400 segmentos censal es mientras que la EN SM 01 tiene cuatro estratos hechos un muestreo independientemente: Tegucigal pa, San Pedro Sula, otro urbano y otro rural.

Por razones relacionadas con el acceso geográfico, costo y menor población, no se realizaron entrevistas en los departamentos de Islas de la Bahía y G racias a Dios.

El trabajo de campo de la EN ESF-01 fue realizado entre el 12 de Febrero y el 19 de A gosto de 2001, para la EN SM -01, el trabajo de campo se realizó entre el 20 de M arzo y el 1 de A gosto de 2001 .

En la EN ESF-01 se identificó un total de 8,578 viviendas con mujeres en edad fértil de 15 a 49 años de edad, el egibles para entrevista, y se entrevistaron 8,578, logrando obtener una tasa de entrevistas completas de 95.6 por ciento.

L as cifras correspondientes a la EN SM -01 son 4,012 viviendas con hombres en edad fértil de 15 a 59 años de edad, de los cuales se entrevistaron a 3,247, resultando en una tasa de entrevistas completas de 79.9 por ciento.

\section{Encuesta de Seguimientos de Mujeres Embarazadas y Mujeres con $\mathrm{N}$ iños menores de un año $(\mathbf{1}, 988)$.}

- Fue realizada por el ministerio de Salud Pública, con organizaciones internacional es como $\mathrm{M}$ anagement Sciences fol $\mathrm{H}$ ealth International ( $\mathrm{MSH}$ ) y Family Health International (FHI).

- Los criterios de selección de mujeres fueron:

- Estar embarazadas y $\mathrm{H}$ aber tenido niños menores de un año de edad al momento de la entrevista.

- $\quad$ Se seleccionaron 2,832 mujeres:

- 995 por estar embarazadas.

- 1,784 por tener un hijo menor de un año.

- 53 por cumplir ambas condiciones.

- Los criterios tomados para evaluar el impacto de la mortalidad infantil:

- Servicios de atención de preparto, parto y posparto.

- Practicas alimenticias.

- M ortalidad y natalidad.

- Los problemas de esta encuesta:

- La muestra fue muy pequeña para estimar indicadores de mortalidad materna.

0 mujeres reentrevistadas.

- negación de embarazos en curso.

- dudas de la situación efectiva de las mujeres.

\section{Encuesta N acional de Salud y N utrición (ENSN)}

- Fue realizada en 1987

- Su objetivo básico: M edir el problema alimentario nutricional.
- La muestra fue de 3,976 vivien das a nivel nacional.

- Se recopiló información socioeconómica, antropométrica para medir el estado nutricional del niño menor de cinco años, morbilidad, inmunizaciones, mortalidad, alimentación infantil, condiciones de salud y paridez en mujeres fértiles, consumo de alimentos en la familia, vivienda, la yodación de la sal en los alimentos, patrón alimentario, estudios hematológico y bioquímicos para caracterizar las anemias.

- $\quad$ En el informe se analiza la relación del valor nutricional de la dieta familiar con al gunas características de la vivienda, de la familia y el estado nutricional del niño.

\section{Encuesta de Ingresos - Gastos y Consumo Real de A limen-} tos (1,978-79)

- La primera de este tipo de encuestas fue realizada en 1967-68 por la Dirección G eneral de Estadísticas y C ensos ( DGEC) Y la Secretaria de Economía y H acienda.

- La de 1978-79 fue ejecutada por el Banco C entral y la DGEC.

- Los objetivos principales de estas encuestas es obtener información sobre

- Los ingresos y las fuentes de estos ingresos.

- G astos en los hogares.

- Patrones de gastos y consumo familiar.

- La muestra fue de 4,225 hogares urbanos y 1,070 hogares rurales a nivel nacional a excepción de Gracias a Dios.

- Las variables tomadas fueron:

$\begin{array}{ll}0 & \text { A ctividad económica. } \\ 0 & \text { Vivienda. } \\ 0 & \text { Ingreso familiar. } \\ 0 & \text { Población. }\end{array}$

\section{Encuestas Continuas Sobre Fuerza de Trabajo (ECSFT)}

- La primera encuesta de este tipo se realizó a principios de 1960 por el M inisterio del Trabajo, otra encuesta referente a la economía familiar fue realizada en 1967-68 por la DGEC.

- La otra fue realizada en los años 1974-75, con una muestra de 4,435 hogares de Tegucigalpa, en contrando un desempl eo abierto de un $8 \%$.

- En 1982 se realizó sobre fuerza de trabajo en Tegucigal pa y San Pedro Sula, por la DGEC y detectó un aumento al desempleo de $15.2 \%$ en Tegucigal pa y $13.7 \%$ en San Pedro Sula, Ia misma se repitió en 1985.

- Objetivos de investigación:

- Investigar las características generales de la población encuestada.

- Condición de actividad de las personas de 10 años y más (ocupación, desocupación e inactividad).

- M edir la PEA, el empleo, subempleo, desempleo e ingresos 
- Con los mismos criterios se realizaron cuatro encuestas continuas de fuerzas de trabajo en 1986-88, a nivel nacional excluyen do I slas de la Bahía y G racias a Dios, realizadas por DGEC. La primera (septiembre de 1986) cubrió 16 ciudades con una muestra de 8,450 viviendas urbanas, la segunda (marzo 1987) en Tegucigalpa, San Pedro Sula, Choluteca, La C eiba y Santa Bárbara.

En septiembre de 1987 se realizó con una muestra de 5,247 viviendas en las áreas rural es de todos los departamentos a excepción de Islas de Bahía y G racias A Dios y la cuarta en septiembre de 1988 se repitió por primera vez tanto a nivel de áreas urbanas como rurales.

\section{Encuesta Agrícola Nacional (EAN)}

Debido a la relación que tiene la producción agrícola y agropecuaria con la Fuerza de Trabajo del país se describen a continuación este tipo de encuestas en forma suscinta:

- El departamento de Encuestas A grícolas dela (DGEC) en 1984 realizó un programa continúo de encuestas agrícolas y agropecuarias a nivel nacional.

- $\quad$ “Estas encuestas se vienen aplicando en el país dos veces por año en los meses de mayo y octubre, a la fecha se han levantado siete encuestas agrícolas nacionales correspondiendo a los años 1984, 1989, 1992, 1995, 1997, 1999 y 2001 " (Entrevista U rrea, A nselmo 2002).

- Estas encuestas constan de cuatro módulos: cultivos anuales, cultivos permanentes, ganadería y tenencia de la tierra.

- $\quad$ El propósito es monitorear y evaluar la recuperación y crecimiento en el sector agrícola (IN E. Encuesta de G ranos Básicos 2001-2002)

\section{Encuesta A grícola N acional 2,001}

El Instituto N acional de Estadística realizó la VII Encuesta A grícola $\mathrm{N}$ acional 2001 con el fin de actualizar la información estadística de este importante sector, los datos correspon den a los cultivos anuales de mayor importancia económica del país.

Esta encuesta genera información sobre el comportamiento reciente de la producción del sector agropecuario nacional, importacionesy exportaciones de productos, datos que sirven para la planificación y toma de decisiones de las instituciones y de los usuarios en general.

En el marco muestral del área no están incorporados los departamentos de G racias a Dios e I slas de la Bahía debido a dificultades de accesi bilidad geográfica y de disponibilidad de material cartográfico en el caso del departamento de $\mathrm{G}$ racias a Dios y la escasa importancia de la agricultura en el departamento de Islas de la Bahía. (INE. Encuesta A grícola N acional 2001-2002).

\section{Encuesta G ranos Básicos 2002}

También realizada por el Instituto $\mathrm{N}$ acional de Estadística
( INE), el levantamiento de la información inició el 11 de junio del 2002 finalizando el 28 de junio del mismo año.

Se realizó con el fin de proporcionar información que permita un conocimiento oportuno a los usuarios del sector agropecuario.

La información se ha organizado en siete secciones que contienen información sobre producción de granos básicos, datos relacionados con la distribución de la superficie sembrada y también sobre el comportamiento histórico de la precipitación pluvial en las diferentes regiones del país.

La encuesta se realizó en 16 departamentos excluyen do los departamentos de Islas de la Bahía y G racias a Dios (IN E. Encuesta de Granos Básicos. 2001-2002).

\section{Encuesta a Líderes $\mathrm{N}$ acionales ( ELN )}

- La A sociación Hondureña de Planificación Familiar (A SH O N PLA FA) realizó en noviembre de 1983 una encuesta a líderes nacionales sobre población y planificación familiar.

- A principios de los años setenta también se había levantado información sobre las opiniones de la élite hondureña acerca del tema de la población, sin embargo, el informe de A SH O N PLA FA no menciona esta encuesta anterior, ni establece comparaciones entre los resultados.

- En 1983 se definió un universo de 932 líderes en los cuales se entrevistó 220 (197 hombres y 23 mujeres), la división entre sectores fue gubernamental, privado y autónomo, donde este último incluye sindicatos, organismos gremiales.

- $\quad$ El $85 \%$ de las entrevistas fueron realizadas en la C apital por ser la sede de los tres poderes del Estado, (7\%) en San Pedro Sula y el otro $11 \%$ en otras ciudades importantes del país.

- En esta encuesta se analizó la población específicamente desde el punto de vista demográfico.

- $\quad$ Siendo los resultados emitidos por los entrevistados sobre problemas socioeconómicos, soluciones y alternativas para mejorar la situación del país, del crecimiento poblacional, nivel de fecundidad, índice de mortalidad infantil, planificación familiar, políticas que el gobierno debe adoptar, crecimiento poblacional migración interna y externa.

- $\quad$ Este tipo de encuesta ya no se realiza en el país.

\section{Encuesta Permanente de H ogares y de Propósitos Múltiples ( EPHPM)}

- Se han venido aplicando regularmente desde 1987 a nivel nacional, dos veces por año, en la mayoría de los años en marzo y septiembre, excepto el año 2000 por el período de transición de la Dirección G eneral de Estadística y C enso a Instituto N acional Estadístico (INE).

- Criterios tomados para realizar la encuesta:

- PEA, PEI, población por sexo y edad, tasa de ocupación, subempleo de la fuerza de trabajo, niveles de ocupación, características de los flujos migratorios internos y exter- 
nos, condiciones de la vivienda en los sectores urbanos, nivel y composición de ingreso.

- A partir de 1988 se redujeron las preguntas destinadas a migración, La cobertura fue en 16 departamentos, excluyendo Gracias a Dios e Islas de la Bahía. En 1989 se incluyó el módulo de preparación y uso del café solicitado por IHCA FE, y la inclusión de las condiciones de vida de los hogares solicitados por el FHIS y el Banco M undial.

Por ser esta encuesta la que se realiza continuamente y hasta la fecha (dos veces al año), la detallamos a continuación con mayor énfasis.

\section{Encuesta Permanente de Hogares y de Propósitos Múltiples ( EPH PM Vigésima Tercera)}

La vigésima tercera encuesta permanente de hogares de propósito múltiples tiene los objetivos siguientes:

1. O btener información sobre las características generales de la población.

2. Estudiar las características de las vivien das en el área urbana y rural.

3. Estimar las tasas de ocupación, sub ocupación y desocupación.

4. Investigar los ingresos de las personas y los hogares, tanto los que son generados por trabajo como los provenientes de otras fuentes ( IN E. Vigésima Tercera EPH, M ayo, 2001 ).

\section{Encuesta Permanente de Hogares y de Propósitos Múltiples (Vigésima Sexta)}

Esta encuesta se realizó a nivel nacional del 10 de septiembre al 10 de noviembre del 2002, en esta oportunidad se levantó información en Islas de la Bahía y G racias a Dios.

$O$ bjetivos de esta encuesta:

- Recolectar información sobre las características general es de la población.

- Identificar la población hondureña migrante y emigrantes.

- Conocer las condiciones del trabajo infantil.

- Investigar las características de las viviendas en el área urbana y rural.

- Determinar las tasas de ocupación, subempleo y desocupación de la población.

- Investigar los ingresos de los hogares.

- Conocer las condiciones y características de la población con discapacidad. ( IN E. Vigésima Sexta EPH, septiembre, 2002 )

E I marco muestral y actualización cartográfica

El marco muestral fue diseñado en 1992, en base al censo de población y vivienda de 1988. Está estructurado en setecientos veinte segmentos distribuidos en dieciséis departamentos.

C ada segmento cuenta con diez a doce viviendas. El total de viviendas de la muestra es de si ete mil doscientos, lo que significa un
$5 \%$ del universo.

La metodología del marco muestral contempla la realización de cuatro réplicas de $25 \%$ cada una para actualizar totalmente la muestra, una vez que se han cumplido cuatro visitas en al guna de las cuatro áreas.

El proceso de rectificación se inició en 1992, con un $25 \%$, no obstante las actualizaciones no se hicieron en forma continua completándose hasta 1995.

C obertura y diseño muestral

Cobertura:

Las características de la población objetivo que investiga EPHPM, se refieren a las personas que residen habitualmente en viviendas particulares. Se excluyen las personas que viven en vivien das colectivas como hoteles, hospitales, cárceles, recintos militares, conventos y otros similares; también se excluyen las personas residentes en embajadas.

La cobertura geográfica de la población objetivo es de alcance nacional a partir del 2002, ya que anteriormente se excluían los departamentos de $\mathrm{G}$ racias a D iose Islas de la Bahía por el difícil acceso y los al tos costos económicos.

Diseño muestral

La muestra tiene como marco muestral la cartografía desarroIlada para los C ensos de Población y Vivienda de 1988 y 2001.

Cuestionario y manuales de capacitación

Las preguntas sobre educación y características de la vivienda son las recomendadas por las naciones unidas a nivel internacional, y además ya ha sido aplicadas varias veces en $\mathrm{H}$ onduras, ya sea a través de los censos de población o de encuestas de investigación social.

Sin embargo, se observa que los mismos podrían ser mejorados en función de conceptualización y con relación a las especificaciones técnicas necesarias para facilitar el Ilenado de la boleta y procesar la información.

Prueba piloto

Debido a que los instrumentos y operaciones no han experimentado grandes cambios en los últimos años, no se efectúa una prueba piloto total.

Levantamiento de datos

El levantamiento comienza en el Distrito C entral con la participación de todos los grupos haciendo el trabajo en un plazo de diez días. Posteriormente, cinco grupos se concentran en San Pedro Sula también por un período de diez días.

A I terminar los mismos se trasladan a la zona noroccidental para cubrir la muestra de Santa R osa de C opán, Santa Bárbara, Yoro y Puerto C ortés.

Los demás grupos son designados a las siguientes zonas: 
A tlántida, Olancho, El Paraíso, Choluteca, Intibucá, C omayagua y la Paz. La gira de estos grupos suelen durar veinticinco días.

U sos de la información

Los resultados de Encuestas de Hogares son usados principalmente por el Banco C entral de H onduras, Ministerio del Trabajo y la U nidad de A poyo T écnico del M inisterio de la Presidencia.

D ebido a los diversos temas investigados con relación a educación y servicios básicos de la vivienda, la misma también es usada a nivel de la Secretaría de Salud y Educación.

U no de los principalesusuariosa nivel internacional es CEPA L que continuamente está sien do estudios de empleo y pobreza a nivel Latinoamericano.

Varios usuarios entrevistados opinaron que es necesario publicar los errores para estar seguros de la confiabilidad de las variables, comentaron que al gunas preguntas en relación al ingreso y a la ocupación secundaria merecían una revisión cuidadosa a fin de mejorar su conceptualización y propósito final de la información.

\section{CONSIDERACIONES FINALES}

1. Las encuestas demográficas obtienen mayor información que los censos, aunque tienen menor cobertura geográfica suministran información detallada sobre temas específicos de la población.

2. Las encuestas se realizan con mayor frecuencia y miden a profundidad niveles y tendencias recientes, siendo un valioso instrumento para capturar datos poblacionales, aunque su periodicidad representa un al to costo económico.

3. La Encuesta Permanente de H ogares de Propósitos M últiples (EPHPM) es el instrumento demográfico de mayor regularidad en su aplicación, proporcionando información actual, útil para una planificación dinámica

4. La mayoría de las encuestas demográficas realizadas en el país no responden a un nivel de desagregación municipal. 